\title{
Influence of Aggregate on the Mechanical Properties of UP Polymer Concrete
}

\author{
Wenxiu Liu ${ }^{1,2, a}$, Jiaming Wu ${ }^{1,2, b}$, Heyi Ge $e^{1,2, c *}$ \\ ${ }^{1}$ Shandong Provincial Key Laboratory of Preparation and Measurement of Building Materials, \\ University of Jinan, Jinan 250022, PR China \\ ${ }^{2}$ School of Material Science and Engineering, University of Jinan, Jinan 250022, PR China \\ aliuwenxiu77@163.com, bfine7frenzy@163.com, cgeheyi@sina.com \\ *Corresponding author: E-mail: geheyi@sina.com
}

Keywords: Aggregate gradation, Polymer concrete, Mechanical property

\begin{abstract}
In this study, polymer concrete (PC) samples were prepared with aggregate, standard sand and unsaturated polyester (UP) resin, the optimal gradation and formula were investigated by orthogonal test. Results show that aggregate gradation is the most important factor in PC mechanical properties. The optimal ratio of coarse aggregate to medium aggregate to fine aggregate was 1:2:1. The optimal contents of aggregate and UP resin were $60 \%$ and $12 \%$, respectively. The maximum compressive and flexural strengths were $65.43 \mathrm{MPa}$ and $21.51 \mathrm{MPa}$, respectively. Aggregates and sands had good distribution in matrix and formed the dense structure observed by metallographic microscope image.
\end{abstract}

\section{Introduction}

Polymer concrete (PC) is a composite material consisting of well-graded inorganic aggregates bonded together by a resin instead of the water and cement binder typically used in traditional cement concretes [1]. Due to its high damping ratio, good vibration resistance, high stiffness weight ration, thermal stability and environmental interference characteristics, PC has been used in machine components and building decoration materials [2]. Unsaturated polyester resin (UP) is one of the binders used in PC providing excellent adhesion to solid particulate fillers, good stiffness, dimensional stability and a high damping ratio [3].

The properties of concrete depend on external and internal factors [4]. External factors include pressure, temperature and construction practices. Internal factors include thickness of concrete, gradation of aggregate, proportions of constituents and mixture properties. The aggregates and standard sand account for a substantial part of PC (volume are about $60 \%$ to $70 \%$ ). The interfacial bond strength from aggregates and polymer resin determines the mechanical properties of PC [5]. PC mechanical properties, such as compressive strength and flexural strength, vary according to the gradation of aggregate. The factor that is usually noticed as the most effective parameter of PC strength is the characteristics of aggregates. In general, aggregate gradation plays a significant role in the mechanical properties of PC.

In this paper, PC samples with different content of aggregate and UP resin were prepared. Effects of aggregate gradation and proportion of components on the mechanical properties of PC were discussed. According to orthogonal $\mathrm{L}_{9}\left(3^{4}\right)$ test, the optimum gradation and formula were determined.

\section{Experimental}

Materials. Aggregates were divided into three grades: coarse aggregate, medium aggregate and fine aggregate. The sands were separated in different particle size with 30 mesh, 50 mesh and 80 mesh by standard sieve, and the ratio of them was 1:2:1 in this work. 198\# unsaturated polyester resin (resin viscosity 0.6-1.2 Pa.S, acid value, 19-27 mg KOH/g) was matrix resin. Methyl-ethyl-ketone peroxide (198\# unsaturated polyester resin and curing agent ratio of 100:2) was curing agent. 
Preparation of PC. Aggregates and standard sands were weighted out in accordance with the proportion. UP resin and curing agent were weighed and mixed well. Mixed the materials from first two steps at room temperature, and finished until the resin binder fully wetting of aggregates and sands. After stirred for approximately 15 minutes, the mixture was poured into $40 \mathrm{~mm} \times 40 \mathrm{~mm} \times 160$ $\mathrm{mm}$ steel tri-mold. Next it was vibrated for $10 \mathrm{~min}$ on the shaking table with 2860 times/min frequency and $10 \mathrm{KN}$ pressure was applied on the surface of the mixture, finally the mold was put in the oven to cure for $2 \mathrm{~h}$ at $90{ }^{\circ} \mathrm{C}$. Then samples were cooled to room temperature. After the process of cutting, grinding flexural strength and compressive strength were tested.

Characterization. The flexural strength and compressive strength test according to the ordinary concrete mechanics performance test standards. The metallographic microscope was used to observe the surface of the PC.

\section{Results and discussion}

ANOVA results in orthogonal test. The investigated levels of each factor were selected depending on experiment results of the single-factor. Independent factors with three variation levels, aggregate content $(55 \%, 60 \%, 65 \%)$, UP resin content $(10 \%, 11 \%, 12 \%)$, aggregate gradation $(1: 1: 2$, $1: 2: 1,2: 1: 1)$ are listed in Table 1 . In the present study, all selected factors were examined using an orthogonal $\mathrm{L}_{9}\left(3^{4}\right)$ test design. The total evaluation results of orthogonal test are presented in Table 2.

Table 1 Factors and levels for orthogonal test

\begin{tabular}{cccc}
\hline NO. & Aggregate content $/ \%$ & UP resin content $/ \%$ & Aggregate gradation \\
\hline 1 & 55 & 10 & $1: 1: 2$ \\
2 & 60 & 11 & $1: 2: 1$ \\
3 & 65 & 12 & $2: 1: 1$ \\
\hline
\end{tabular}

Table 2 Analysis of $\mathrm{L}_{9}\left(3^{4}\right)$ test results

\begin{tabular}{cccccc}
\hline NO. & $\begin{array}{c}\text { Aggregate } \\
\text { content }\end{array}$ & $\begin{array}{c}\text { UP resin } \\
\text { content }\end{array}$ & $\begin{array}{c}\text { Aggregate } \\
\text { gradation }\end{array}$ & $\begin{array}{c}\text { Compressive } \\
\text { strength/MPa }\end{array}$ & $\begin{array}{c}\text { Flexural } \\
\text { strength/MPa }\end{array}$ \\
\hline 1 & 1 & 1 & 1 & 54.53 & 15.07 \\
2 & 1 & 2 & 2 & 58.66 & 18.33 \\
3 & 1 & 3 & 3 & 57.39 & 17.90 \\
4 & 2 & 1 & 3 & 56.70 & 16.54 \\
5 & 2 & 2 & 1 & 55.96 & 15.91 \\
6 & 2 & 3 & 2 & 65.43 & 19.51 \\
7 & 3 & 1 & 2 & 61.62 & 14.84 \\
8 & 3 & 2 & 1 & 53.81 & \\
9 & 3 & 3 & 3 & 54.13 & \\
$\mathrm{~K}_{1 \mathrm{C}}$ & 56.86 & 57.62 & 54.77 & & \\
$\mathrm{~K}_{1 \mathrm{~F}}$ & 17.10 & 17.17 & 15.27 & & \\
$\mathrm{~K}_{2 \mathrm{C}}$ & 59.36 & 56.14 & 61.90 & & \\
$\mathrm{~K}_{2 \mathrm{~F}}$ & 17.99 & 16.36 & 19.91 & & \\
$\mathrm{~K}_{3 \mathrm{C}}$ & 56.62 & 58.98 & 56.07 & & \\
$\mathrm{~K}_{3 \mathrm{~F}}$ & 16.64 & 17.04 & 16.54 & & \\
$\mathrm{R}_{\mathrm{C}}$ & 3.09 & 2.84 & 7.14 & & \\
$\mathrm{R}_{\mathrm{F}}$ & 1.35 & 0.81 & 4.64 & & \\
\hline
\end{tabular}

Influence of aggregate gradation on mechanical properties. It can be found in Table 2 that the maximums of PC strengths were obtained when aggregate content was $60 \%$, UP resin content was $12 \%$ and aggregate gradation was 1:2:1. The compressive and flexural strengths were $65.43 \mathrm{MPa}$ and 
21.51 $\mathrm{MPa}$, respectively. According to $\mathrm{K}_{1 \mathrm{C}}$ and $\mathrm{K}_{1 \mathrm{~F}}$, compressive (flexural) strength had a slight improvement when aggregate content increased from $55 \%$ to $60 \%$, and then decreased by aggregate content increased to $65 \%$. Compressive (flexural) strength first decreased when content of UP resin increased to $11 \%$, and reached a maximum until content of UP resin increased to $12 \%$ according to $\mathrm{K}_{2 \mathrm{C}}$ and $\mathrm{K}_{2 \mathrm{~F}}$. When aggregate gradation was $1: 2: 1$, strengths of $\mathrm{PC}$ reached a maximum. When coarse aggregate content was high $(2: 1: 1)$, resin was easy to settle down during preparation process. This phenomenon would leave cavity in the sample resulting in poor mechanical properties. Besides, high content of fine aggregate (1:1:2) also adversely affected the strength of PC. This is primarily caused by fine aggregate will increase the viscosity of mixture and caused poor distribution of fillers. So, appropriate aggregate gradation is the key point to PC property.

According to $\mathrm{R}_{\mathrm{C}}$ and $\mathrm{R}_{\mathrm{F}}$ values, the factors influence the flexural strength and compressive strength of PC listed in a decreasing order as follows: aggregate gradation, aggregate content, UP resin content. So gradation of aggregate was the dominant factor of flexural and compressive strengths.

The microstructure of PC. The metallographic microscope image of PC (60\% aggregate, $12 \%$ resin and 1:2:1 gradation) is shown in Fig. 1. It can be seen that aggregates and sands were distributed homogeneously in matrix and formed the dense structure. There was basic no hole in PC. It demonstrates that appropriate aggregate gradation is the key point to PC property.

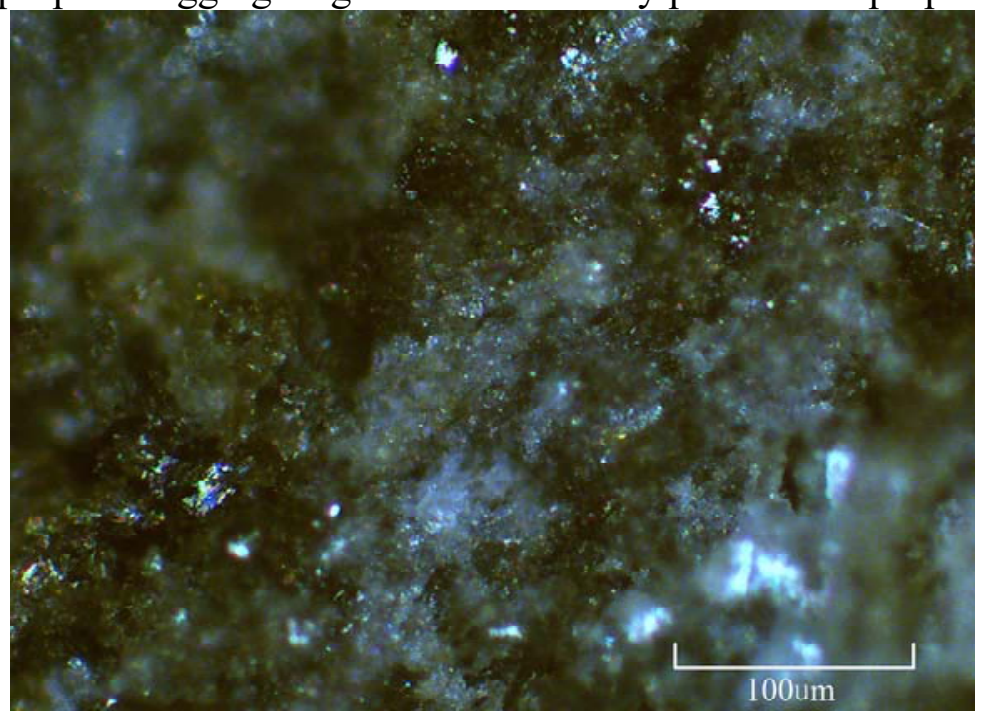

Fig. 1 Metallographic microscope

\section{Conclusions}

PC samples were prepared by adding different gradation and content of aggregate in UP resin to investigate the influence on mechanical properties. According to a orthogonal $\mathrm{L}_{9}\left(3^{4}\right)$ test, when aggregate content was $60 \%$, UP resin content was $12 \%$ and aggregate gradation was $1: 2: 1$, the PC compressive and flexural strengths reached to maximum $(65.43,21.51 \mathrm{MPa})$. Furthermore, mechanical properties of PC were predominant by gradation of aggregate. The metallographic microscope image shows that aggregates and sands were distributed homogeneously in matrix and formed the dense structure.

\section{Acknowledgements}

This work is supported by Special plan for independent innovation and achievements transformation of Shandong Province, China (Grant No. 2014ZZCX05302). 


\section{References}

[1] H. Haddad, M. A. Kobaisi: Composites: Part B, Vol. 43 (2012) No.8, p. 3061.

[2] Y. Qin, Z. Q. Huang, H. Wang and Z. X. Huang: New Building Materials, (2007) No.7, p. 75. (In Chinese)

[3] A. M. Ferreira, M. S. Ribeiro, A. T. Marques: International Journal of Mechanics and Materials in Design, Vol. 1 (2004) No.2, p. 143.

[4] G. Amir, J. Ehsan, N. Yunus, et al: Social and Behavioral Sciences, (2012) No.53, p. 440.

[5] Y. Wang, H. Q. Sun, W. Y. Du, W. B. Li: China Concrete and Cement Products, (2014) No.5, p. 12. (In Chinese) 